\title{
Sutureless skin closure for pacemaker implantation: comparison with subcuticular suture
}

\author{
DAVID PITCHER* \\ M.B., M.R.C.P. \\ Department of Cardiology, Plymouth General Hospital, Plymouth, Devon
}

\begin{abstract}
Summary
In 51 patients undergoing pacemaker implantation a trial was carried out to compare the results of sutureless skin closure using a new product (Op-Site skin closures) with skin closure using a continuous subcuticular absorbable (Dexon) suture. Incisions closed with Op-Site skin closures healed as well as those closed with subcuticular suture but were associated with less frequent and less extensive erythema around the skin edges one week after the procedure. They were quicker, simpler and cheaper to apply than the suture material used in this study.
\end{abstract}

KEY WORDS: cardiac pacing, skin closure, healing.

\section{Introduction}

The implantation of a cardiac pacemaker is a relatively minor surgical procedure, usually performed using local anaesthesia. Using strict aseptic technique, the procedure is associated with a low rate of infection $(0-5 \%)$ despite the presence of foreign material in the tissues (Lemire, Morin and Dobell, 1975; Linschoten et al., 1973; Schaudig et al., 1977). Nevertheless, when serious infection does occur conservative treatment is rarely effective and the pacemaker usually has to be removed and implanted elsewhere (Morgan et al., 1979). Conventional skin closures may encourage infection; sutures passing through the skin carry both epidermis and bacteria into the subcutaneous tissues (Gillman and Penn, 1956; Gillman, 1958). Furthermore, many patients await the removal of such sutures with anxiety. Over a 3-year period, the author had used an absorbable subcuticular suture (Dexon) after pacemaker implantation. Subjective assessment indicated that this technique was acceptable to patients and was rarely associated with serious infection.

Recent studies showed a new product for sutureless skin closure to be effective in closing incisions in thoracic and abdominal surgery (Westaby, 1980;

*Present address: Cardiac Department, Bristol Royal Infirmary, Bristol BS2 8HW.
Eaton, 1980). Op-Site skin closures had the advantage over conventional techniques of being quicker and easier to apply with better cosmetic results. Whilst subcuticular sutures may avoid some of the potential dangers of conventional skin sutures, trauma to the skin edges during insertion may cause local bruising leading to delayed healing and wound infection. A sutureless technique would avoid this problem, together with any inflammatory response to the suture material itself.

A trial was therefore undertaken to compare the results of skin edge apposition using sutureless $(\mathrm{Op}-$ Site) skin closures and subcuticular (Dexon) sutures in patients undergoing pacemaker implantation.

\section{Patients and methods}

Fifty-one consecutive patients ( 28 male, 23 female, aged 50-89 years) undergoing uncomplicated implantation of a permanent pacing system or elective replacement of a pacemaker generator were included in the trial. All procedures were carried out by the same operator (D.P.). New pacing systems were implanted via a single subclavicular incision, a Vitatron Helifix endocardial electrode being introduced by direct puncture of the subclavian vein using a modification of the technique described by Batson (1973). All patients received either a Vitatron S3121 or a Telectronics 183 generator implanted in the prepectoral region. These two generators are of approximately equal size, weight and shape.

Patients whose procedures were regarded as 'complex' were excluded from the trial. These procedures were those involving more than one incision, usually required for an alternative route of electrode insertion, or in patients with pre-existing skin necrosis. The results in these patients were assessed separately and will be presented in this report.

In patients undergoing uncomplicated pacemaker implantation or generator replacement, the subcutaneous tissues were closed using standard techniques with 2 layers of continuous plain catgut sutures. 
TABLE 1. Details of patients included in the trial

\begin{tabular}{lcl}
\hline \multicolumn{1}{c}{$\begin{array}{c}\text { Method of skin } \\
\text { closure }\end{array}$} & $\begin{array}{c}\text { Sutureless (Op-Site) } \\
\text { skin closure }\end{array}$ & $\begin{array}{l}\text { Subcuticular (Dexon) } \\
\text { suture }\end{array}$ \\
\hline Number of patients & 25 & 26 \\
New pacing systems & 7 & 10 \\
Generator replacements & 18 & 16 \\
Age (yrs) (mean \pm s.d.) & $73 \cdot 6 \pm 7 \cdot 5$ & $74 \cdot 6 \pm 9 \cdot 1$ \\
Length (mm) of skin incision (mean \pm s.d.) & $49 \cdot 3 \pm 6 \cdot 5$ & $50 \cdot 2 \pm 7 \cdot 3$ \\
\hline
\end{tabular}

Patients were allocated to either sutureless skin closures or subcuticular sutures according to a predetermined randomization. In patients allocated to Op-Site, a $10 \times 14 \mathrm{~cm}$ Op-Site skin closure was cut to the required width using sterile scissors and applied so as to achieve good apposition of the skin edges. In patients allocated to subcuticular sutures these were inserted using 2/0 Dexon in standard fashion. The time taken to insert the subcuticular suture was noted in each case. After skin closure by either technique, the wound was covered by a dry gauze dressing. Antibiotic prophylaxis was not used in patients with uncomplicated pacemaker implantations.

Patients undergoing generator replacement were usually discharged home the following day; patients with new pacing systems remained in hospital at least 5 days. All patients were reassessed one week after pacemaker insertion or replacement. Any subjective observations were noted. The length of the incision was measured and the size of any surrounding inflammation was also measured. In the event of any discharge from the wound, a swab was taken for bacteriological culture. Antibiotics were prescribed at the discretion of the reviewing doctor. Op-Site skin closures were removed at this visit. All patients were reviewed a second time one month after pacemaker insertion. Any further symptoms or residual signs of inflammation or infection were noted.

Wound healing in patients whose skin was closed with Op-Site was compared with that in those whose skin was closed using subcuticular sutures. Fisher's exact test was used to assess the statistical significance of the results.

\section{Results}

There were no important differences between the 2 groups of patients with respect to age, length of skin incision and type of procedure (Table 1 ). The average time taken to insert the subcuticular sutures was $6 \frac{1}{2}$ min; application of skin closures took only a few seconds.

Incisions in both groups of patients healed well. One week after pacemaker insertion, a larger proportion $(16 / 25)$ of the Op-Site group had incisions healed without any surrounding erythema than did the suture group (10/26), but this difference was not statistically significant. Extensive erythema, involv ing the entire length of the incision or extending $\mathrm{mm}$ or more from the skin edges, was present in significantly $(P=0.003)$ fewer patients in the Op-Siter group $(1 / 25)$ than in the group in whom sutures were used $(10 / 26)$.

One infection occurred: a patient in the sutur $\varepsilon_{\infty}^{\infty}$ group developed fever and distension of the pace $\omega$ maker pocket 3 days after pacemaker implantation despite apparently good skin healing. Altered bloot was aspirated from the pacemaker pocket. Staphylo ${ }_{\mathbb{Q}}^{\top}$ coccus aureus was isolated from this aspirate an from blood cultures. Fortunately the infection reฏ sponded well to systemic treatment with flucloxacillin and fusidic acid together with repeated aspiratfong of the infected haematoma and local instillation sot gentamicin to the pacemaker pocket. Slight sergivis discharge from the wound edges occurred in a furthe? 2 patients in the suture group and in 3 patients in the Op-Site group. No organisms were isolated from the discharge in any of these patients. All were given course of antibiotics, and in all subsequent wound healing progressed uneventfully. Small superficia haematomata developed and subsequently dis charged spontaneously in one further patient in the suture group and in 2 patients in the Op-Site group one of whom was taking warfarin. Slight skin overlaps occurred in one patient whose skin was closed with. Op-Site, but this was not associated with any. inflammation or infection. At one month all incisions. were healing well. Extensive erythema persisted in only one patient, whose skin had been closed witb sutures.

Patient acceptance of both skin closure technique was good. One patient in the Op-Site group found the green non-adhesive edges of the skin closure uncoms fortable and attempted to remove them herself $N$ causing partial detachment of the skin closure in the process. In subsequent patients the edges of the skinf closures were trimmed off immediately after applica $x$ tion and there were no further reports of discomfort

Eight patients were excluded from the trial as theik pacing procedures involved more than one incision ${ }^{+}$ In the first of these 'complex' cases sutures were used but Op-Site skin closures were used for the other $7 \overrightarrow{\mathbb{D}}$ 
One of these patients developed a superficial wound infection after her skin closures were removed prematurely by a district nurse. The wound healed quickly following superficial toilet and systemic antibiotic therapy. In all other patients with complex pacing procedures, good wound healing was achieved using Op-Site skin closures.

\section{Discussion}

Effective skin closure after any surgical procedure requires apposition of the skin edges for sufficient time to allow healing to take place. Movement between the skin edges and excessive tension must be avoided (Eaton, 1980), but mild tension distributed evenly produces a stronger skin repair at an early stage (Forrester, Zederfeldt and Ahren, 1970). OpSite skin closures appear to meet these requirements. The results of this study show this material to be at least as good as subcuticular Dexon sutures in achieving satisfactory healing after pacemaker implantation. In this small study I could not have expected to show any difference in infection rates between the two techniques since infection is an infrequent complication of pacemaker insertion in this unit, as elsewhere (Lemire, Morin and Dobell 1975; Linschoten et al., 1973; Schaudig et al., 1977). The sutureless skin closures had the advantage of being quicker and simpler to apply and were associated with less frequent, less extensive erythema around the incision. They are also cheaper than the suture material used (skin closures $0.61 \mathrm{p}$ each, suture material $0.96 \mathrm{p}$ each). The greater erythema associated with subcuticular sutures may have been due to an inflammatory response to the suture material, or possibly to skin-edge trauma caused by suture insertion. It was not associated with any demonstrable bacterial infection.

This study has shown Op-Site skin closures to be effective, and it has emphasized two important points with regard to their use. Firstly, the green nonadhesive edges are best removed immediately after application of the skin closure. Otherwise they may cause mild discomfort and encourage the patient to interfere with the skin closures despite written or verbal instructions to the contrary. This is particu- larly important in pacemaker patients, many of whom are elderly and may not follow such instructions easily. Secondly, if selected patients are to be discharged into the community with skin closures in place, district nurses and general practitioners must be informed as to their nature and purpose so that inappropriate premature removal of the skin closures (as occurred in one of the patients excluded from the trial) is avoided.

With this proviso, Op-Site skin closures appear to be a simple, cheap and effective method of skin closure for patients undergoing pacemaker implantation.

\section{Acknowledgments}

I wish to thank Drs J. Cowie and A. J. Marshall for permission to include patients in this study. I am grateful to Mr P. Mackenzie of Smith \& Nephew Ltd for providing the skin closures.

\section{References}

BATSON, G.A. (1973) A simplified technique for permanent transvenous endocardial pacing. Lancet, ii, 597.

EATON, A.C. (1980) A controlled trial to evaluate and compare a sutureless skin closure technique (Op-Site Skin Closure) with conventional skin suturing and clipping in abdominal surgery. British Journal of Surgery, 67, 857.

ForRester, J.C., ZeDerfeldT, B.H. \& AHREN, C. (1970) Tapeclosed and sutured wounds: a comparison by tensiometry and scanning electron microscopy. British Journal of Surgery, 57, 729.

GILLMAN, T. (1958) Healing of cutaneous abrasions and of incisions closed with sutures or plastic adhesive tape. Medical Proceedings, $4,751$.

Gillman, T. \& PenN, J. (1956) Studies on the repair of cutaneous wounds with reference to epidermal reactions to sutures and the pathogenesis of carcinoma in scars. Medical Proceedings, 2, Suppl., 121.

LemiRe, G.G., MORIN, J.E. \& Dobell, A.R.C. (1975) Pacemaker infections: a 12-year review. Canadian Journal of Surgery, 18, 181.

LiNSCHOTEN, H., MEIJNE, N.G., MELLINK, H.M. \& OVERDIJK, A.D. (1973) Pacemaker implantation and infection. Journal of Cardiovascular Surgery, 14, 126.

Morgan, G., Ginks, W., Siddons, H. \& Leatham, A. (1979) Septicaemia in patients with an endocardial pacemaker. American Journal of Cardiology, 44, 221.

SChaudig, A., Zimmerman, M., Thurmayr, R. \& Beyer, J. (1977) Komplikationen der schrittmachertherapie. Internist (Berlin), 18, 25.

WESTABY, S. (1980) Evaluation of a new product for sutureless skin closure. Annals of the Royal College of Surgeons of England, 62 , 129.

(Accepted 27 August 1982) 